\title{
Alternative solar-battery charge controller to improve system efficiency
}

\begin{abstract}
When the heat losses sense is adopted for some solar system and power conversion components in residential building application, it is benefit to use those losses, that are consequently acts inversely on system efficiency, as a useful energy to modify the performance of that system instead of dissipated. In terms of efficiency of solar-battery charge controller, power dissipation is always associated with every power component and the losses would be either results from switching and conducting components or from the OFF state of the main switching component. This paper proposed a high efficiency solarbattery charge controller as an alternative to the main solar charge controller in most conventional residential solar system, the concept of the proposed controller circuit based on using the off state energy duration as well as that power losses coming from the main switching component and complexity of the conventional controller, and using this energy in a separate current path to energize an auxiliary battery or directly load used to cool or even to ventilate the system components causing in improving its performance. This will leads to electronic circuit with low losses as a compared with the conventional charge controller to be a part of the integrated and automated building solar system, the design has an algorithm based on some environment parameters like sun Irradiance and weather temperature, this algorithm seems to be inversely calculated because its start from the value of full charge battery voltage. A Simulink Matlab simulator is attempted in the simulation phase of this research as well as an experimental data has been collected to verify the circuit function and energy saving goal.
\end{abstract}

Keyword: Battery charge controller; Buck converter; MPPT; Power electronicslosses; PV solar analysis; Solar cell parameters 